\section{Bestrahlung nach Operation? Sofort!}

Die Anzeichen dafür, dass eine sofortige Radiotherapie nach radikaler Prostatektomie bei Patienten mit fortgschrittenem Prostatakarzinom nützlich ist, mehren sich - sogar, wenn der PSA-Spiegel unter die Nachweisgrenze gesunken ist, wie nun ein deutsches Team berichtet.

L okales Versagen nach radikaler Prostatektomie ist häufig, wenn das Prostatakarzinom über die Kapsel hinausgewachsen ist. Zwei randomisierten Studien zufolge profitieren Patienten von einer adjuvanten Radiotherapie. Gilt dies auch für Patienten, deren PSA-Spiegel nach der Operation unter die Nachweisgrenzen gefallen sind? Eine multizentrische deutsche Arbeitsgruppe ist dieser Frage nachgegangen.

385 Patienten wurden im Rahmen einer Phase-III-Studie randomisiert. 193 von ihnen sollten dirket nach radikaler Prostatektomie eine Radiotherapie erhalten, die anderen nur beobachtet werden. Alle Patienten hatten Tumoren im Stadium pT3 pN0. 78 Patienten wurden von der Behandlung ausgeschlossen, da der PSASpiegel postoperativ nicht unter die Nachweisgrenze sank. Von den verbliebenen 307 Patienten erhielten 34 keine Bestrah- lung, obwohl sie dem Radiotherapie-Arm zugelost waren; umgekehrt bekamen fünf Patienten in der Kontrollgruppe doch eine Radiotherapie. Somit wurden insgesamt 114 Patienten mit 60 Gy in 30 Fraktionen bestrahlt, 154 wurden lediglich beobachtet. Der primäre Endpunkt war biochemisch progressionsfreies Überleben.

Nach fünf Jahren war diese Rate in der Radiotherapie-Gruppe signifikant höher. Sie lag bei 72 gegenüber $54 \%$, was einer Hazard-Ratio von 0,53 entsprach. Eine Multivarianz-Analyse ergab, dass neben der Radiotherapie ein präoperativer PSASpiegel von $<10 \mathrm{ng} / \mathrm{ml}$ und das Tumorstadium (pT3a/b vs. pT3c) das Behandlungsergebnis signifikant günstig beeinflussten. Die Rate schwerer Spättoxizitäten vom Grad 3/4 lag bei 0,3\%.

Fazit: Eine adjuvante Radiotherapie bei Prostatakarzinomen im Stadium pT3 mit

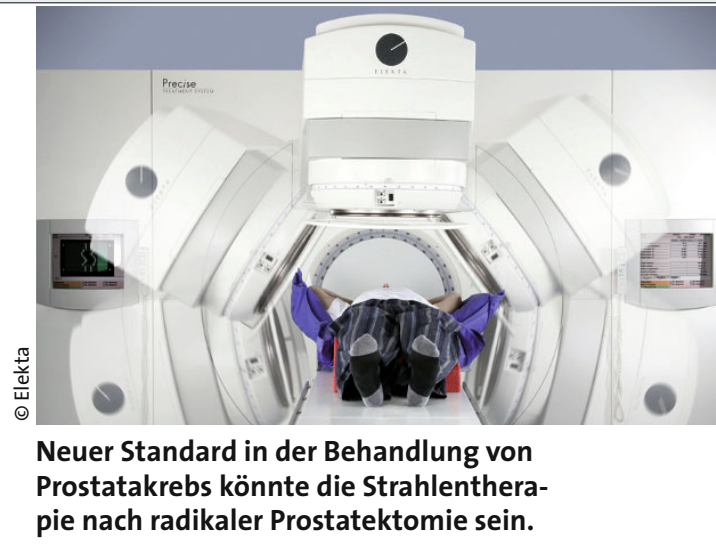

postoperativ undetektierbarem PSA-Spiegel senkt das Rsiko für biochemische Progression signifikant. Weiteres Follow-up ist zwar nötig, um die Auswirkungen auf metastasenfreies und Gesamt-Überleben herauszufinden. Doch in einem begleitenden Kommentar schreiben drei USExperten bereits, dass sich die sofortige postoperative Radiotherapie wohl als neuer Therapiestandard etablieren wird. be

Wiegel T et al. Phase III postoperative adjuvant radiotherapy after radical prostatectomy compared with radical prostatectomy alone in $\mathrm{PT} 3$ prostate cancer with postoperative undetectable prostate-specific antigen: ARO 96-02/AUO AP 09/95. J Clin Oncol 2009; 27: 2924-30. - Comment: Thompson $I M$ et al. Is there a standard of care for pathologic stage T3 prostate cancer? Ibidem 2898-9.

\title{
Widerstandstraining gegen Fatigue
}

\begin{abstract}
Fatigue zählt zu den häufigsten Beschwerdebildern im Zusammenhang mit einer Strahlentherapie. In einer kanadischen Studie mit Prostatakarzinom-Patienten wurde der Effekt eines mehrwöchigen supportiven Widerstandstrainings oder von Aerobic-Übungen auf das therapiebedingte Fatigue-Syndrom, die Lebensqualität und körperliche Fitness untersucht.
\end{abstract}

\footnotetext{
P rostatakrebs-Patienten unter Strahlentherapie entwickeln in 60-80\% der Fälle ein oft über die Therapie hinaus anhaltendes Fatigue-Syndrom. Fatigue ist verbunden mit psychologischem Leid und reduzierter Lebensqualität. In einer prospektiven, randomisierten und kontrollierten Studie wurden die möglichen Vorteile eines 24-wöchigen Widerstandstrainings oder Aerobic-Trainings gegenüber der konventionellen Betreuung untersucht. Beurteilt wurden: Fatigue, Lebensqualität, körperliche Fitness, Muskel-FettVerteilung, PSA-Wert, Lipid-Werte sowie Testosteron- und Hämoglobin-Spiegel bei
}

Prostatakarzinom-Patienten unter einer Radiotherapie.

Die Teilnehmer wurden in drei etwa gleich große Gruppen aufgeteilt: Widerstandstraining, Aerobic-Training oder konventionelle Betreuung. Primärer Endpunkt war die Fatigue, gemessen anhand der FACT (Functional Assessment of Cancer Therapy)-Fatigue-Skala. Beide Trainingsprogramme verbesserten kurzfristig den Fatigue-Score. Patienten, die am Widerstandstraining teilnahmen, profitierten zudem von einer erhöhten Lebensqualität, waren besser trainiert, wiesen günstigere Tri- glyzeridwerte und Testosteronwerte auf. Das Widerstandstraining führte im Gegensatz zu Aerobic auch zu einer länger anhaltenden Linderung der Fatigue. Verglichen mit konventioneller Betreuung zeigte sich beim Widerstandstraining aber eine geringere Reduktion im PSA-Wert. Die klinische Bedeutung dessen ist unklar.

Fazit: Beide Trainingsarten sind geeignete supportive Maßnahmen zur kurzfristigen Linderung von radiotherapiebedingten Fatigue-Symptomen bei Prostatakarzinom-Patienten. Das Widerstandstraining wirkt zudem auch längerfristig Fatigueassoziierten Symptomen entgegen und trägt zu einer Steigerung der Lebensqualität der Patienten bei.

zi

Segal MC et al. Randomized controlled trial of resistance or aerobic exercise in men receiving radiation therapy for prostate cancer. J Clin Oncol 2008; 27: 344-51. 